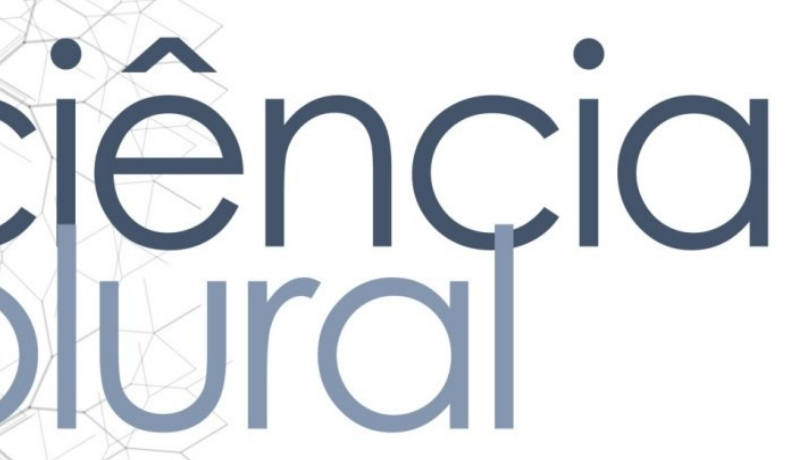

\title{
VIABILIDADE DE SUBSTITUIÇÃO DE SUCOS INDUSTRIALIZADOS POR SUCOS NATURAIS NA ALIMENTAÇÃO ESCOLAR
}

\section{Viability of ready-to-drink juices substitution to natural juices in school meals}

Mikaella Hayanne Medeiros dos Santos • Acadêmica do Curso de Nutrição da Faculdade de Ciências da Saúde do Trairi da Universidade Federal do Rio Grande do Norte - UFRN. E-mail: mikaellajs@hotmail.com

Dinara Leslye Macedo e Silva Calazans • Nutricionista. Doutora em Administração. Especialista em Vigilância Sanitária. Professora do Curso de Nutrição da Faculdade de Ciências da Saúde do Trairi da Universidade. E-mail: dinaraleslye@yahoo.com.br

Taiana Brito Menêzes Flor • Nutricionista graduada pela Universidade Federal do Rio Grande do Norte. Mestre em Saúde Coletiva pela Universidade Federal do Rio Grande do Norte. Escola Agrícola de Jundiai. Restaurante Universitário. E-mail: taiana_bm@yahoo.com.br

Fábio Resende de Araújo • Nutricionista. Doutor em Administração. Professor do Curso de Nutrição da Faculdade de Ciências da Saúde do Trairi da Universidade Federal do Rio Grande do Norte - UFRN. E-mail: fresende87@gmail.com

Autor responsável pela correspondência:

Taiana Brito Menêzes Flor. Nutricionista graduada pela Universidade Federal do Rio Grande do Norte. Mestre em Saúde Coletiva pela Universidade Federal do Rio Grande do Norte. Escola Agrícola de Jundiai. Restaurante Universitário. E-mail: taiana_bm@yahoo.com.br 


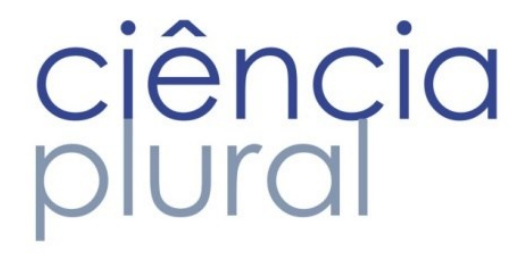

RESUMO

Introdução: O Programa Nacional de Alimentação Escolar (PNAE) faz parte da Política Pública que preconiza a promoção de alimentação saudável e restrição de alimentos ultraprocessados, tendo o nutricionista papel fundamental na garantia de suas diretrizes. A promoção de hábitos alimentares saudáveis em idade precoce (infância e adolescência) favorece a redução de DCNT na vida adulta, sendo a escola ambiente privilegiado para isto. Objetivos: 0 objetivo deste estudo foi verificar a viabilidade técnica, operacional e econômica da substituição dos sucos prontos servidos atualmente na alimentação escolar em uma escola pública federal por sucos naturais, com base nas diretrizes dispostas pela PNAE. Métodos: Trata-se de um estudo de viabilidade realizado no Restaurante Universitário da Escola Agrícola de Jundiaí, vinculada à Universidade Federal do Rio Grande do Norte. Foram analisadas três grandes variáveis: econômicas, que avaliam o custo para produção dos sucos; operacionais, que visam verificar os pontos mais relevantes a serem considerados para que se tenha efetividade de produção de sucos; e técnicas, que analisam a qualidade nutricional e sanitária dos sucos. Resultados: Para todas as variáveis estudadas a oferta do suco natural comparativamente ao suco industrializado se apresentou como melhor escolha. Economicamente o suco industrializado apresentou custo unitário maior que $200 \%$ em relação ao suco natural. Em relação a variável técnica, nutricionalmente, o suco natural apresentou maior percentual de fibras, vitaminas e minerais, além de um menor teor de sódio. Não houve limitações estruturais, nem de equipamentos e utensílios ou mudanças significativas no fluxo de produção da unidade com a inserção do suco natural. Conclusão: Torna-se, então, comprovada a viabilidade de substituição dos sucos prontos industrializados pelo suco natural favorecendo a melhor utilização dos recursos públicos e a oferta de uma alimentação nutricionalmente mais adequada, contribuindo para efetivação do PNAE enquanto Politica Pública.

Palavras Chave: Política de saúde. Alimentação escolar. Alimentos industrializados.

\section{ABSTRACT}

Introduction: The National School Food Program is a Brazilian government policy which regulates school meals, recommending healthy food promotion and restriction of ultraprocessed food and the nutricionist has an important role in the guarantee its guidelines. The healthy food habits promotion in early age (childhood and adolescence) favors reduction of chronic disease in adult life, being the school a privileged place to do this. Objective: The aim of the study was to verify the economical, operational and technician viability of ready-todrink juice substitution served nowadays on school meals in a public school to natural juices, based on guidelines proposal by Brazilian policy. Methods: A feasibility study was done in an university restaurant of Jundiai Farm School, linked to Rio Grande do Norte Federal University. Three big variables were analysed: economicals, which evaluates the cost of juices production; operational, which aims to verify to most important points to be considered to have effectiveness in juice production; and technicians, which analyses the juices' nutritional quality and sanitary. Results: All variables studied showed the offer of natural juice comparing to ready-to-drink juice as the best choice. Economically, the ready-to-drink juice had a unit cost of more than $200 \%$ related to natural juice. Related to technical variable, the natural juice presented higher nutritional quality with fibers, vitamins and minerals, and lower sodium content. There were no structural limitations; equipment and utensils were sufficient. The production flow is suitable for the natural juice production. Conclusions: The viability of ready to drink juices subtitution was comproved favoring an use of public resources in the best way and the offer food nutritionally more appropriate, contributing to National School Food Program effectiveness as a public policy.

Keywords: Health Policy. School Feeding. Industrialized Foods. 


\section{ciência \\ plural}

\section{Introdução}

A alimentação escolar é hoje um desafio a ser cumprido em muitos municípios do país. Nessa perspectiva, o Programa Nacional de Alimentação Escolar (PNAE) faz parte da política de governo que orienta a concretização do Direito Humano à Alimentação Adequada (DHAA) e a promoção da alimentação saudável nas escolas. O PNAE atende a todos os alunos matriculados nas escolas públicas, da creche ao ensino médio, inclusive educação de jovens de adultos e, por esse motivo, é considerado um dos grandes programas de alimentação escolar de todo o mundo que não somente do Brasil1.

Os recursos financeiros desse programa são repassados pelo Fundo Nacional de Desenvolvimento da Educação (FNDE), tem caráter suplementar e deverão ser destinados a compra de gêneros alimentícios visando ofertar refeições adequadas às necessidades nutricionais médias da clientela e seguras em termos higiênico-sanitários. Para tanto, é designado o gerenciamento e a responsabilidade técnica da oferta da alimentação escolar ao profissional nutricionista², que deverá, dentre outras atribuições, garantir a melhor escolha dos alimentos que irão compor para cada tipo de refeição.

A escolha dos alimentos diz respeito também à proteção da saúde, principalmente, àquela relacionada aos fatores que contribuem para a prevenção das doenças crônicas não transmissíveis (DCNT), que representam, no Brasil, grande problema de saúde pública, responsável por $72 \%$ dos óbitos e associadas a incapacidades funcionais que comprometem a qualidade de vida da população ${ }^{3}$.

Neste sentido, a escola desponta como lócus privilegiado de ações de promoção à saúde, de prevenção aos fatores de risco e proteção dos escolares. O PNAE determina alimentos que são proibidos ou restritos na oferta da alimentação escolar, entre os quais se destacam os ultraprocessados. Os alimentos prontos ou semiprontos para consumo, tais como os sucos industrializados, são considerados restritos na alimentação escolar, isto é, não são proibidos desde que o valor gasto com esse tipo de alimento não ultrapasse $30 \%$ do valor repassado para compra do total de insumos ${ }^{2}$.

Paradoxalmente ao estímulo a alimentação saudável, em muitas escolas ainda é ofertada uma alimentação inadequada, com forte presença de alimentos industrializados e ultraprocessados, que durante sua fabricação - visando a dilatação da vida de prateleira - sofrem perdas consideráveis do seu valor nutricional, 


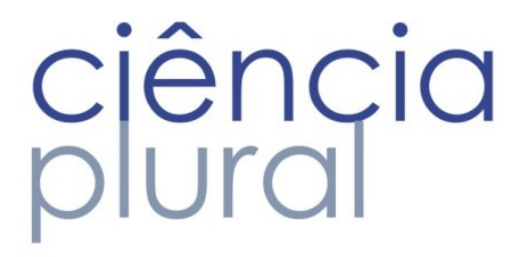

corroborando com um novo padrão alimentar, que atinge estudantes brasileiros de todos os níveis econômicos e regiões, marcado pela redução do consumo de alimentos in natura e aumento desses primeiros.

Este cenário provocou inquietações profissionais durante a realização do estágio curricular em Gestão da Alimentação Coletiva do curso de graduação em Nutrição, a partir da observação in loco de uma frequente oferta de sucos industrializados ultraprocessados na alimentação escolar. Haja vista que a proibição deste alimento não é expressa, mas recomendada pelo PNAE, aspectos gerenciais que envolvem a produção e distribuição das refeições e avaliação do custo-benefício dos gêneros alimentícios adquiridos, podem não estar harmonicamente disponíveis para a efetivação da oferta restrita do mesmo.

Assim, a fim de promover uma alimentação que esteja alinhada com os pressupostos das Politicas de Saúde voltadas à Alimentação e Nutrição, o objetivo deste estudo é verificar a viabilidade técnica, operacional e econômica da substituição dos sucos prontos por sucos naturais na alimentação escolar ofertada em uma escola pública federal, com base nas diretrizes dispostas pelo PNAE.

\section{Referencial Teórico}

O Programa Nacional de Alimentação Escolar (PNAE), em funcionamento no Brasil desde a década de 50, mundialmente conhecido como merenda escolar, é reconhecido como uma politica pública sustentável e que contribui comprovadamente para o desenvolvimento de crianças e adolescentes melhorando seu rendimento escolar e favorecendo a segurança alimentar, por meio da oferta de uma alimentação escolar adequada 1 .

Esta alimentação oferecida é ainda um dos importantes mecanismos de proteção de riscos à saúde, como a obesidade que afeta contemporaneamente jovens e adultos com consequências negativas à sua qualidade de vida; ao tempo que estimula a formação de hábitos alimentares saudáveis ${ }^{7}$, como 0 incentivo ao consumo adequado de frutas e hortaliças.

O estímulo ao consumo frequente de frutas, também é ditado pela Portaria $1.010^{5}$ que estipula estratégias para se alcançar a alimentação saudável na escola como o aumento da oferta e promoção do consumo de frutas, legumes e verduras. Também procura atingir essa promoção de saúde a partir de capacitação dos profissionais que participam na produção da merenda escolar definindo estratégias e restringindo alimentos não saudáveis a ser ofertados dentro da escola de forma a facilitar a escolha de opções saudáveis nos lanches. 


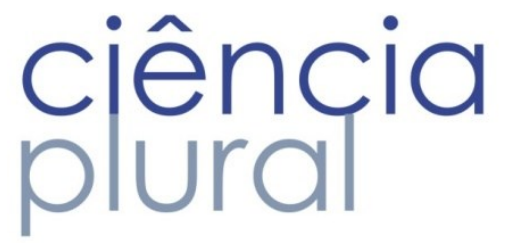

Esta portaria é um marco para promoção da alimentação saudável e considera todo o contexto que envolve a alimentação escolar sejam esses sociais, nutricionais ou mesmo pedagógicos. Dentre as outras considerações da portaria, de fato, esta corrobora com a afirmação de que a boa nutrição dos escolares confere uma melhor qualidade de vida e de saúde o que contribui efetivamente para reversão do perfil epidemiológico do brasileiro, ratificando a alimentação como parâmetro imprescindível no âmbito escolar ${ }^{5}$.

Contudo, esta realidade ainda não é alcançada em muitas escolas, existindo ainda oferta de alimentação inadequada com excesso de alimentos ultraprocessados ${ }^{3}$. 0 Guia Alimentar para a população Brasileira, define como alimentos ultraprocessados "aqueles feitos à base de substâncias extraídas de alimentos, derivados de constituintes de alimentos, ou feitas à base de matérias orgânicas em laboratório"4.

Apesar de origem multifatorial, a alimentação inadequada está entre os fatores de risco mais relevantes e a adolescência é a fase de vida caracterizada por mudanças constantes decorrentes de inúmeros fatores como o meio em que este está inserido na sociedade, os princípios, a cultura e os costumes regionais além dos hábitos alimentares ${ }^{6}$. Por isso, esse período é considerado fundamental na formação de hábitos alimentares saudáveis e na qualidade de vida na fase adulta, especialmente considerando que este comportamento previne as DCNT.

Dados da Pesquisa Nacional de Saúde do Escolar ${ }^{3}$ que analisou estudantes adolescentes mostrou que 0 consumo semanal de alimentos considerados não saudáveis, como os ultraprocessados foi consideravelmente maior do que a média na população (16,4\%) na Região Nordeste com adolescentes entre 13 e 15 anos de idade $(20,2 \%)$. Os alimentos classificados como não saudáveis por essa pesquisa também foram maiores quanto se tratou de guloseimas e salgados fritos e os percentuais de legumes e verduras estiveram abaixo da média para todos entre 13 e 17 anos, tendo o Nordeste o menor percentual de consumo destes em relação às outras regiões. Esses dados evidenciam o perfil de má alimentação nesta fase da vida e, por isso, a importância de se voltar políticas de saúde à alimentação saudável.

Considerado um dos maiores programas de alimentação de todo o mundo, o PNAE traz em seus princípios a alimentação e nutrição muito bem fundamentada, pois estes consideram, além da adequação nutricional, outros fatores como a igualdade, cultura alimentar e as tradições de cada região ${ }^{1} \mathrm{e}$, sabendo da complexidade de hábitos alimentares na adolescência e da de alta prevalência de morte por DCNTs, que incluem doenças no coração, no aparelho respiratório, assim como diabetes, obesidade e os mais diversos tipos de câncer, torna-se crucial a mudança de hábitos alimentares, especialmente nesta fase da vida, trazendo-a para uma melhor qualidade de vida decorrente de diminuição das DCNTs ${ }^{7}$. 
Metodologia

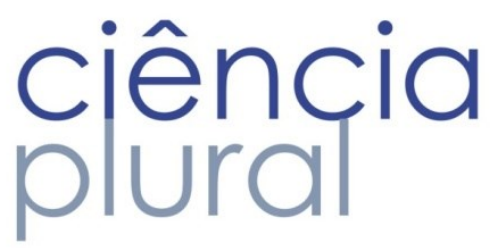

Trata-se de um estudo de viabilidade realizado na Escola Agrícola de Jundiai (EAJ) - Unidade acadêmica especializada em Ciências Agrárias da Universidade Federal do Rio Grande do Norte (UFRN), durante 0 estágio curricular supervisado em Gestão em Alimentação Coletiva do Curso de Nutrição da Faculdade de Ciências da Saúde do Trairi/UFRN, no período de julho a outubro de 2016.

A EAJ conta com o apoio de uma Unidade de Alimentação e Nutrição (UAN) para fornecer alimentação dos alunos inseridos regularmente nesta instituição: o Restaurante Universitário, que atende cerca de 800 refeições/dia (desjejum, almoço e jantar) destinadas a estudantes da escola, e cerca de 600 lanches/dia (colação, lanche da tarde) destinados exclusivamente aos alunos da educação básica, beneficiários do PNAE, residentes na escola. Funciona todos os dias e dispõe de quadro de funcionários terceirizados, sendo os nutricionistas e gestores da Unidade servidores públicos. Adquire gêneros alimentícios para produção das refeições por meio de licitação na modalidade de pregão eletrônico.

Os dados foram coletados in loco, por meio de observações diretas, medições e análise documental utilizando-se um roteiro semiestruturado com aspectos determinantes de cada variável. As variáveis (Quadro 1) foram definidas a partir dos fatores a serem considerados na seleção de alimentos e preparações no planejamento de cardápio ${ }^{8}$ e das atividades que competem ao nutricionista no exercício de suas atribuições em Unidades de Alimentação e Nutrição e na Alimentação Escolar ${ }^{9}$.

Quadro 1: Aspectos analisados por variável utilizada no estudo de viabilidade - Macaíba/RN - 2016

\begin{tabular}{|l|l|}
\hline Variáveis & Aspectos analisados \\
\hline \multirow{5}{*}{ Econômicas } & $\begin{array}{l}\text { Valor unitário dos gêneros alimentícios adquiridos } \\
\text { Valor unitário de aquisição de materiais descartáveis utilizados } \\
\text { Valor/hora de trabalho da mão-de-obra } \\
\text { Valor unitário do material de limpeza utilizado } \\
\text { Consumo de energia elétrica para armazenamento e produção dos sucos } \\
\text { Consumo de água para produção e higienização }\end{array}$ \\
\hline \multirow{5}{*}{ Operacionais } & $\begin{array}{l}\text { Tempo disponível/gasto para produção de sucos } \\
\text { Mão-de-obra despendida }\end{array}$ \\
& $\begin{array}{l}\text { Espaço físico disponível para armazenamento e produção } \\
\text { Fluxograma de produção }\end{array}$ \\
& Cronograma de abastecimento de gêneros alimentícios \\
& Disponibilidade de descartáveis do setor \\
& Estado de conservação e manutenção dos equipamentos de produção e estoque \\
\hline & $\begin{array}{l}\text { Pesos brutos (PB), líquidos (PL) e fatores de correção (FCç) utilizados para a } \\
\text { preparação dos sucos in natura } \\
\text { Composição nutricional dos sucos elaborados } \\
\text { Informação nutricional contida nos rótulos dos sucos prontos } \\
\text { Exequibilidade e adequação do cumprimento das BPF na produção dos sucos }\end{array}$ \\
\hline
\end{tabular}

Fonte: autoria própria com base em Vieira e Japur (2012) e Brasil (2005). 


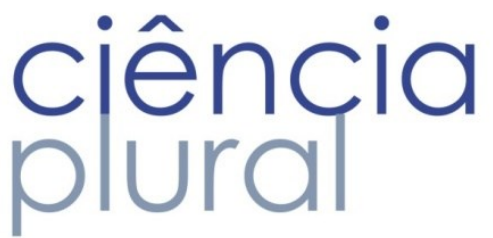

Foram analisadas três grandes variáveis: econômicas, que avaliaram o custo para produção dos sucos; operacionais, que visaram verificar os pontos mais relevantes a serem considerados para que se tenha efetividade de produção de sucos; e técnicas, que analisaram a qualidade nutricional e sanitária dos sucos.

A análise econômica partiu do custo direto de produção com gêneros alimentícios obtidos a partir da verificação de Fichas Técnicas de Preparação $(\mathrm{FTP})^{8}$ do local, as quais consideraram uma proporção padrão de polpas de frutas e água mineral de 1:3 com percentual de açúcar de 10\% para todos os sabores e porção de $180 \mathrm{ml}$. Estes foram somados aos demais custos indiretos descritos a seguir. Os preços dos itens (gêneros alimentícios, descartáveis e material de limpeza) para determinação do custo unitário foram extraídos da consulta ao sistema de controle orçamentário ${ }^{10}$ próprio da universidade onde foi possível ter acesso aos pregões vigentes e contrato com empresa terceirizada de mão-de obra, sendo verificado neste último os valores pagos em salários e a jornada de trabalho. 0 cálculo do consumo de energia elétrica considerou: tempo de utilização dos equipamentos no preparo, dispêndio de energia durante 0 armazenamento dos gêneros alimentícios e distribuição do suco, em khw, por tipo de equipamento e taxa de consumo, de acordo com a recomendação da Companhia Energética do Rio Grande do Norte (COSERN) ${ }^{11}$. 0 consumo da água encanada utilizada se baseia na relação proposta pela Associação Brasileira de Normas Técnicas (ABNT) ${ }^{12}$ de $25 \mathrm{~L}$ por refeição completa, sendo assim feita a conversão para lanches (5:1), de acordo com Conselho Federal de Nutrição (CFN) ${ }^{9}$ e considerada a estrutura tarifária da Companhia de Águas e Esgotos do Rio Grande do Norte (CAERN) ${ }^{13}$. Para a água mineral estimada para a produção dos sucos foi considerado seu valor unitário de compra e per capita (130 ml) estabelecido pelo local.

Quanto aos aspectos que envolvem a variável operacional, foi acompanhada a produção já existente dos sucos naturais no local para outra refeição (almoço) e verificado: espaço físico disponível, a fim de analisar suficiência do espaço para manejo e dinâmica de produção dos sucos; adequação do dimensionamento de equipamentos, móveis e utensílios utilizados no preparo; o estado de conservação e manutenção dos equipamentos de produção e estoque foi verificado, realizando-se o levantamento do histórico de quebras e/ou paradas na produção por falta de manutenção e tempo de reposição e reparo. 0 fluxograma de produção foi elaborado, para verificar a dificuldade ou facilidade do serviço e a adequação com a marcha avante recomendada 8 . 0 cronograma de abastecimento de gêneros alimentícios foi analisado de modo a verificar a garantia da quantidade de gêneros alimentícios para produção do suco suficiente para cobrir a demanda. Para a oferta de sucos naturais considerou-se ainda a entrega do açúcar, da agua mineral e disponibilidade de descartáveis do setor. 


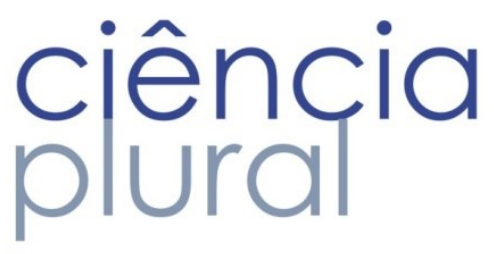

Para análise da variável técnica partiu-se da FTP8 (Peso Limpo=Peso Bruto=70g; Fator de Cocção=0) dos sucos naturais realizando-se a análise química nutricional de cada sabor (polpa de fruta) e, posteriormente, obtendo-se a média aritmética de composição de nutrientes, com base em tabelas de composição de alimentos 14,15. Quanto aos sucos prontos, foi verificada a informação nutricional dos rótulos das embalagens de $200 \mathrm{~mL}$ dos sabores disponíveis no local e realizado o mesmo procedimento para obtenção da média aritmética que os dos sucos naturais. Ainda nesta variável, realizou-se também análise qualitativa higiênico-sanitária a partir da observação da conformidade dos requisitos de boas práticas, do estado de conservação, periodicidade de limpeza dos equipamentos de preparo e estoque, e possibilidade de contaminação cruzada conforme resolução sanitária vigente ${ }^{16}$.

A análise da viabilidade se deu a partir da comparação do custo-refeição dos sucos naturais e o valor de compra/estoque dos sucos prontos, seguido de discussão sobre pontos positivos e negativos relacionados à operacionalização do preparo dos sucos naturais em relação aos sucos prontos e, por fim, a verificação da qualidade nutricional e sanitária de cada tipo de suco, evidenciando seu custo-efetividade.

\section{Resultados e Discussão}

As diferenças entre os sucos analisados foram relevantes vista as três dimensões de variáveis propostas na metodologia. O suco natural obteve melhores resultados em comparação ao suco pronto na maioria dos aspectos estudados o que levou a ser considerada a sua viabilidade de oferta.

Foi visto que para o preparo de sucos naturais do almoço são gastos cerca de 40 minutos, inferindo-se que, desta maneira, seriam gastos em média 35 minutos para produção de 300 sucos por 2 auxiliares de cozinha, equivalente a um turno de distribuição. Na tabela 1 é possível verificar a síntese do custo-refeição do suco natural a ser servido nos lanches em comparação ao preço unitário do suco pronto industrializado. 0 custo com material de limpeza não foi contabilizado ao custo unitário final de cada suco, por não ser representativo, considerando o custo total por refeição. 


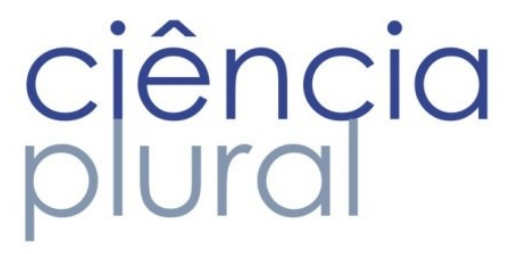

Tabela 1: Custo-refeição do suco natural comparado ao custo unitário suco pronto. Macaíba/RN - 2016.

\begin{tabular}{lll}
\hline Itens para produção de suco & Sucos naturais & Sucos prontos \\
Sucos de polpa & $0,33^{*}$ & - \\
Mão-de-obra & 0,04 & - \\
Descartáveis & 0,03 & - \\
Energia & 0,01 & $0,00^{* *}$ \\
Água & 0,01 & - \\
Total de custo unitário & $\mathbf{0 , 3 7}$ & $\mathbf{0 , 8 7}$ \\
\hline
\end{tabular}

Fonte: Elaboração própria, 2016. Nota: *média aritmética dos sabores de sucos natural já preparados; **Energia para armazenamento de sucos industrializados.

O custo de produção enfatiza que, o suco natural proveniente de polpa de fruta é mais viável economicamente do que o suco pronto industrializado, considerando que o percentual de açúcar dos sucos pode ainda ser diminuído ou, pelo menos, variado entre os sabores e influenciar mais positivamente no custo de unitário dos sucos naturais.

Esta medida vem, inclusive, ao encontro das recomendações da Organização Mundial de Saúde, expressas em Normas Técnicas do PNAE, dentre as quais a limitação do consumo de açúcar, que objetivam contribuir para garantia do crescimento e o desenvolvimento adequados, da promoção da saúde e da prevenção das doenças relacionadas à alimentação, como a redução do risco de obesidade e outras alterações metabólicas ${ }^{17}$.

No entanto, mesmo a análise de custo indicando o suco natural como mais barato, é fato que este ainda ultrapassou o limite orçamentário repassado pelo FNDE de $R \$ 0,30$ por aluno. Este fato é recorrente nos estudos que analisam os custos com os cardápios do PNAE, na maioria dos municípios brasileiros o custo excede 0 valor repassado pelo Governo Federal, indicando a necessidade de complementação do recurso pelas demais esferas e instâncias de gestão competentes, o que pode acontecer nas escolas federais, como a escola agrícola, contribuindo para aumento desse valor fazendo com que o suco possa permanecer na merenda2; a insuficiência do valor do repasse ao programa é a principal crítica realizada pelos conselhos de alimentação escolar ${ }^{18}$.

De outro modo, uma das estratégias para diminuição de custos pode ser alcançada a partir do estabelecido na Lei no $11.947^{19}$ que disponibiliza o Programa Dinheiro Direto na Escola (PDDE) para compra direta de produtos da agricultura familiar (AF) e que pode contribuir diretamente para melhora da qualidade dos alimentos que são oferecidos na merenda. Sabendo da localidade (rural) onde a escola agrícola se encontra e, levando em conta a cultura alimentar da região (nordeste), a possibilidade de compra de alimentos naturais 


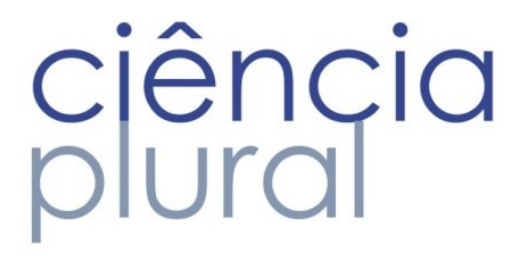

como frutas frescas, ou mesmo a polpa de fruta natural advindas da AF, com um valor mais justo torna-se maior e favorece o cumprimento da cota de $30 \%$ de aquisição da agricultura familiar.

Quanto à variável operacional, não houve dificuldade na operacionalização decorrente do espaço físico ou estrutura disponível. A sala de preparo é exclusiva para sucos e sobremesas, dotada de visores que facilitam a supervisão do nutricionista, possui $12 \mathrm{~m}^{2}$ e dispõe de bancadas que ocupam o comprimento de $1 / 4$ da sala estando em conformidade com o recomendado para setores de pré-preparo de lanches em UANs de pequeno e médio porte $\left(0,035 \mathrm{~m}^{2} \times \text { CMA/turno }\right)^{20}$, sendo a CMA a capacidade máxima de atendimento de refeições por turno de produção.

O dimensionamento de equipamentos, móveis e utensílios foram suficientes à produção de sucos de polpa de frutas para os lanches. Não são necessários mais utensílios para distribuição dos sucos, além dos usuais, já disponíveis em capacidade suficiente no local. O liquidificador (industrial basculante) possibilita produzir 54 litros de sucos por turno (300 porções), sendo ligados 4 vezes até a quantidade total.

O abastecimento de polpa de fruta e açúcar ao estoque é feito quinzenalmente, e água mineral, semanalmente, ocorrendo o controle de estoque alinhado à demanda, realizado sob supervisão do gestor, e evitando a deterioração de alimentos, prazos de validade vencidos, excesso ou falta de itens e até mesmo desvios, conforme salientado na Nota Técnica do FNDE, onde o controle de estoque de alimentos, a o permitir realizar uma previsão mais real da aquisição dos gêneros alimentícios e maior segurança no acompanhamento do fluxo dos alimentos adquiridos, é tido como mecanismo gerencial destinado à promoção do direito humano à alimentação adequada no âmbito do PNAE 21 .

O fluxograma de produção se mostrou efetivo, sem o comprometimento de outras operações ou do preparo de refeições seguintes. Essa afirmação se dá especialmente pelo fato de que a produção acompanhada in loco (operação/tempo) foi de uma quantidade de suco cerca de duas vezes maior do que a que seria necessária à oferta no lanche, portanto, a produção de sucos naturais nos lanches é favorável.

A operacionalização das etapas de produção é um ponto crucial na análise e foi um fator corroborativo com a produção de sucos, pensando que a oferta de uma alimentação mais saudável não afetará também 0 fluxo de produção da unidade. Além disso, o PNAE afirma que as Entidades Executoras (EEs) devem disponibilizar recursos financeiros para que a unidades de alimentação possuam qualidade nas refeições, inclusive em relação ao espaço físico, equipamentos, móveis e utensílios ${ }^{1}$. 
Em relação à variável técnica, o aspecto nutricional pode ser verificado de forma comparativa na Tabela 2. Os nutrientes avaliados foram os determinados pelo PNAE como cumprimento de parte (15\%) das necessidades nutricionais dos escolares atendidos. Dentre as diferenças nutricionais mais significativas evidenciou-se a presença de fibras do suco natural, assim como uma menor quantidade de sódio e presença de outros minerais e vitaminas, os quais não estão presentes no suco industrializado (pronto para consumo).

Tabela 2: Análise nutricional dos sucos naturais e sucos prontos (industrializados).

Macaíba/RN - 2016.

\begin{tabular}{l|l|l|l|l|l|l|l|l|l|l}
\hline $\begin{array}{l}\text { Tipo de } \\
\text { suco }\end{array}$ & $\begin{array}{l}\text { Energia } \\
\text { (Kcal) }\end{array}$ & $\begin{array}{l}\text { Carb } \\
(\mathbf{g})\end{array}$ & $\begin{array}{l}\text { G.Total } \\
(\mathbf{g})\end{array}$ & $\begin{array}{l}\text { Prot } \\
(\mathbf{g})\end{array}$ & $\begin{array}{l}\text { FibTot } \\
(\mathbf{g})\end{array}$ & $\begin{array}{l}\text { Vit A } \\
(\mathbf{R E})\end{array}$ & $\begin{array}{l}\text { Vit C } \\
(\mathbf{m g})\end{array}$ & $\begin{array}{l}\mathbf{N a} \\
(\mathbf{m g})\end{array}$ & $\begin{array}{l}\text { Ca } \\
(\mathbf{m g})\end{array}$ & $\begin{array}{l}\text { Fe } \\
(\mathbf{m g})\end{array}$ \\
\hline Natural & 96,69 & 24,96 & 0,00 & 0,00 & 0,18 & 0,00 & 13,5 & 5,02 & 3,41 & 0,03 \\
\hline Pronto & 119,00 & 29,00 & 0,00 & 0,00 & 0,00 & 0,00 & 0,00 & 8,00 & 0,00 & 0,00 \\
\hline
\end{tabular}

Fonte: elaboração própria (2016) com base em Philiphi, 2013; TACO, 2011. Nota: carboidrato (carb); gordura total (g. total); proteínas (prot); fibras totais (fib. tot.); vit (vitamina).

A Estratégia Global da $\mathrm{OMS}^{22}$ recomenda restringir o sódio consumido e, por isso, tem-se a importância da substituição dos sucos industrializados, exatamente pela oferta de sódio que pode vir a superar as recomendações máximas diárias ao final do dia. Um estudo recente ${ }^{23}$ que analisou o teor de sódio nas preparações de escolas públicas do município de Natal/RN relatou que apenas metade das escolas se apresentava com um valor de sódio na merenda escolar conforme o preconizado pelo PNAE o que demonstra ainda maior apreensão em manter baixos teores de sódio a serem ofertados na merenda escolar, inclusive na Escola Agrícola.

Apesar de o suco não poder ser considerado uma porção de fruta como a estabelecida pelo Guia alimentar para a população brasileira ${ }^{4}$, contribui para a cota semanal de $200 \mathrm{~g}$ recomendado pelo PNAE e tornase uma opção melhor, em termos nutricionais, do que o suco industrializado (ultraprocessado, pronto a beber), pois provém de frutas naturais as quais são recomendadas também pela Estratégia Global22 e que são importantes para manutenção de baixos teores de sódio na merenda escolar, além de aproximar a população de escolares ao consumo de formas in natura de alimentos.

O achado corrobora ainda com a importância de fortalecer políticas públicas voltadas a alimentação e nutrição sendo as escolas o principal local onde a realidade atual do país - o processo de transição nutricional, considerando, principalmente, a mudança drástica nos hábitos alimentares que resultaram na extinção da fome e surgimento do sobrepeso e obesidade - pode ser mudada ${ }^{24}$. A estratégia global fortalece esta afirmativa ao 


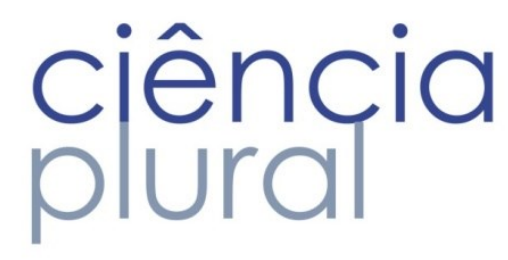

recomendar que as políticas alimentares incentivem a promoção da saúde pública e facilitem a adoção pela população de uma alimentação saudáve|22.

Outro ponto a favor do suco natural foi evidenciado pela Empresa Brasileira de Pesquisa Agropecuária25 ao afirmar sobre a riqueza de vitaminas e minerais que fazem a qualidade nutricional das frutas e hortaliças e a importância destas para a saúde e longevidade, visando ainda a proteção contra DCNTs. E, pode-se ainda destacar, a importância destes alimentos para a prevenção de carências nutricionais. A adequação alimentar é um requisito no planejamento da merenda escolar e, portanto, a inserção de frutas e derivados desta no cardápio pode contribuir para esta adequação vista a qualidade nutricional já mencionada, o que condiz com 0 papel do nutricionista do PNAE na elaboração de cardápios da merenda escolar².

Quanto à análise do aspecto higiênico-sanitário, os setores de armazenamento, pré-preparo e preparo, e distribuição dos lanches apresentou um percentual médio de adequação de $90 \%$, estando em conformidade com as boas práticas preconizadas pela vigilância sanitária.

Os pontos críticos para o controle sanitário na produção de sucos são efetivos, como as polpas de frutas são armazenadas em freezer exclusivo, não estando concomitantemente com outros alimentos e é conservada uma média de temperatura de $-20^{\circ} \mathrm{C}$; as bancadas e equipamentos são higienizados com detergente neutro e álcool a $70 \%$ antes e depois do preparo dos sucos, estando em bom estado de conservação; as polpas são lavadas ainda na embalagem apenas em água corrente antes de serem abertas.

Em relação aos hábitos de higiene dos manipuladores, apesar de apresentar um percentual satisfatório de conformidade, as mãos dos colaboradores são lavadas frequentemente, porém não seguem exatamente procedimento conforme instrução de trabalho afixada em pia exclusiva para o fim próxima a sala de preparo. Contudo isto pode ser resolvido enfatizando o treinamento do pessoal e exigindo o cumprimento das medidas preventivas de Boas Práticas de Manipulação descritas na Resolução RDC 216/2006 ${ }^{16}$. Em contrapartida, os sucos prontos (industrializados) possuem menos perigos de contaminação decorrente da tecnologia de sua embalagem tetra park que não possibilita erros de manipulação decorrente de má conduta higiênica dos colaboradores.

A segurança dos alimentos também é um requisito disposto pelo PNAE e, por isso, a análise qualitativa sanitária é imprescindível neste trabalho. Garantir a qualidade da alimentação escolar é tarefa multifatorial para o nutricionista e, por isso, é importante manter a equipe que participa desta atividade, sejam merendeiras ou diretores, muito bem instruída para que erros de manipulação, por exemplo, sejam evitados. 


\section{ciência \\ plural}

\section{Conclusões}

A oferta de sucos naturais é um modo de incentivar bons hábitos alimentares e, consequentemente, contribuir para a redução de sobrepeso e obesidade na vida adulta, realidade atual da população brasileira. Além disso, a escola é o local onde a população mais passível de aprendizado se encontra e, portanto, tem-se o compromisso de fazer da alimentação saudável um processo educativo.

Frente os resultados, a viabilidade de substituição dos sucos prontos industrializados, restritos pelo PNAE, pelos sucos de frutas naturais, produzidos no local, foi comprovada. Sugere-se que este processo seja iniciado a fim de melhor utilizar recursos públicos e ofertar um alimento nutricionalmente mais adequado.

Sugere-se que estudos como este possam ser reproduzidos em outras escolas do país, com vistas apoiar à gestão das politicas de saúde voltadas à alimentação e nutrição nas escolas públicas.

\section{Referências}

1. Brasil. Fundo Nacional de Desenvolvimento da Educação. Secretaria de Educação a Distância. 2nd ed. MEC FNDE SEED, editor. Mec, Fnde, Seed. Brasília; 2008. 112 p.

2. Brasil. Fundo Nacional de Desenvolvimento da Educação. Resolução CD/FNDE No 26 de 17 de junho de 2013. Dispõe sobre o atendimento da alimentação escolar aos alunos da educação básica no âmbito do Programa Nacional de Alimentação Escolar . Brasília: Ministério da Educação, 2017.

3. Instituto Brasileiro de Geografia Estatística. Pesquisa nacional de saúde escolar: 2015.rio de janeiro: IBGE; 2016.

4. Brasil. Guia alimentar para a população brasileira. 2. ${ }^{2} e d$. Brasilia:Ministério da Saúde; 2014.

5. Brasil. Minstério da Saúde. Portaria Interministerial No 1.010de 8 de maio de 2006. [Internet]. Brasilia:Ministério da Saúde; 2006. Disponível em: http://crn3.org.br/legislacao/doc/1.010_2006.pdf

6. World Health Organization. Nutrition in adolescence: issues and challenges for the health sector [Internet]. Issues in Adolescent Health and Development. Geneva; 2005. 32 p. Available from: http://apps.who.int/iris/bitstream/10665/43342/1/9241593660_eng.pdf

7. Organização Pan-Americana da Saúde. Doenças crônico-degenerativas e obesidade: estratégia mundial sobre alimentação saudável , atividade física e saúde. Brasília:Organizaçaõ Pan-Americana de Saúde; 2003.

8. Vieira MNCM, Japur CC. Gestão da qualidade na produção de refeições. Rio de Janeiro: Guanabara; 


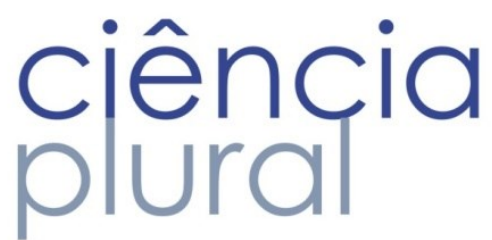

9. Brasil. Conselho Federal de Nutrionista. Resolução CFN no 380 de 28 de dezembro de 2005. Dispõe sobre a definição das áreas de atuação do nutricionista e suas atribuições, estabelece parâmetros numéricos de referência, por área de atuação, e dá outras providências [Internet]. 2005 . Disponivel em: http://www.cfn.org.br/novosite/pdf/res/2005/res380.pdf.

10. Universidade Federal do Rio Grande do Norte. Superintendência de Informática. SIPAC - Sistema Integrado de Patrimônio, Administração e Contratos [Internet]. 2011 [acessado em 22 set. 2016]. Disponível em; https://docs.info.ufrn.br/doku.php?id=suporte:sipac:visao_geral

11. Rio Grande do Norte. Companhia Energética do Rio Grande do Norte (COSERN )[Internet]. [citado em 10 set. 2016]. disponível: http://servicos.cosern.com.br/residencial-rural/Pages/Baixa Tensão/aprenda-a-ler-seumedidor.aspx.

12. Associação Brasileira de Normas Técnicas. NBR 5626: Instalação predial de água fria. rio de Janeiro: ABNT; 1998.

13. Rio Grande do Norte. Companhia dee Águas e Esgotos do Rio Grande do Norte. Estrutura tarifária $\begin{array}{lllll}\text { [Internet]. } & \text { [citado } & 24 & \text { maio } & \text { 2016]. }\end{array}$ http://si.caern.com.br/gsan/exibirConsultarEstruturaTarifariaPortalCaernAction.do

14. Philiphi ST. Tabela de composição de alimentos: suporte para decisão nutricional. 4. ${ }^{a}$ ed. rio de Janeiro: Manole; 2013.

15. Universidade Estadual de Campinas(Unicamp). Nucleo de Estudos e Pesquisas em alimentação. Tabela brasileira de composição de alimentos(TAPO) [Internet]. Campinas: UNICAMP; 2011. Disponível em: http://www.unicamp.br/nepa/taco/

16. Brasil. Ministerio da Saúde. Agencia Nacional de Vigilancia Sanitaria. Resolução RDC 216 de 15 de setembro de 2004. Diário oficial [da]Republica Federativa do Brasil, Brasilia, DF, 2004.

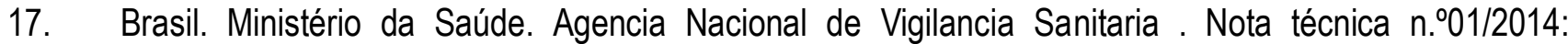
vigilância e monitoramento das infecções relacionadas à assistência à saúde (IRAS) e resistência microbiana (RM) em serviços de saúde. Brasília: Ministério da Saúde; 2014.

18. Silva MV da S, Danelon MS. Conselhos de Alimentação Escolar (CAEs): análise dos pareceres conclusivos sobre a execução do Programa Nacional de Alimentação Escolar (PNAE). Segurança Aliment e Nutr. 2013;20(1):122-35.

19. Brasil. Lei no 11.947 de 16 de junho de 2009. Diario Oficial [da] Republica Federativa do Brasil, Brasilia, DF, 2009.

20. Sant'ana HMP. Planejamento Físico-funcional de Unidades de Alimentação e Nutrição. 2. ${ }^{a}$ ed. São Paulo: Rubio; 2012.

21. Brasil. Ministério da Educação. Nota técnica n 5002/ COSAN/CGPAE/DIRAE [internet]. disponível em: 


\section{ciência \\ plural}

https://www.fnde.gov.br/fndelegis/action/UrlPublicasAction.php?acao=getAtoPublico\&sgl_tipo=NTC\&num_ato=0 0005002\&seq_ato $=000 \& v\left|r \_a n o=2016 \& s g\right|$ orgao=FNDE/MEC\&cod_modulo=9\&cod_menu=940

22. World Health Organization .Global Strategy, on diet, physical activity and health [Internet]. Geneva; 2004. Available from: http://medcontent.metapress.com/index/A65RM03P4874243N.pdf

23. Pereira EB. Avaliação do teor de sódio na alimentação de estudantes de escolas públicas em NatalRN[monografia]. Natal, RN; 2016.

24. Brasil. Política Nacional de Alimentação e Nutrição.Brasilia: Ministério da Saúde; 2013. 86 p.

25. Moretti CL, Oliveira DA, Vieira JV, Nascimento WM, Timm CA, Macedo A, et al. A importância nutricional das hortaliças. Brasília: EMBRAPA; 2012. 